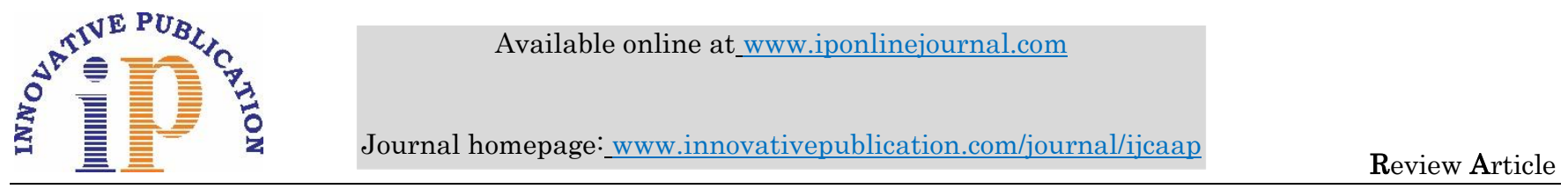

\title{
A review on pain and its signals modulation by body
}

\author{
Rashmi B.R ${ }^{1 *}$, Ebrahim N.K.C ${ }^{2}$
}

${ }^{1}$ Lecturer, ${ }^{2}$ Senior Lecturer, ${ }^{1,2}$ Dept. of Physiology, ${ }^{1}$ A.J Institute of Allied Health Sciences, Kuntikan, Mangalore, Karnataka, India, ${ }^{2}$ Faculty of Medicine, Quest international university, Perak, Malaysia

\begin{abstract}
Pain is an uncomfortable feeling that tells you something may be wrong. It can be steady, throbbing, stabbing, aching, pinching, or described in many other ways. Sometimes, it's just a nuisance, like a mild headache. Other times it can be debilitating. Pain can bring about other physical symptoms, like nausea, dizziness, weakness or drowsiness. It can cause emotional effects like anger, depression, mood swings or irritability. Pain can change your lifestyle and impact your job, relationships and independence. Pain is classified as either acute or chronic. Acute pain is usually severe and short-lived, and is often a signal that your body has been injured. Chronic pain can range from mild to severe, is present for long periods of time, and is often the result of a disease that may require ongoing treatment. When we sense pain, we pay attention to our bodies and can take steps to fix what hurts. Pain also may prevent us from injuring a body part even more. If it didn't hurt to walk on a broken leg, a person might keep using it and cause more damage. If your throat is really sore, you'll probably go to the doctor, who can treat the infection if you have one. Pain itself often modifies the way the central nervous system processes pain, so that a patient actually becomes more sensitive and gets more pain with less provocation. This is called "central sensitization." (And there's peripheral sensitization too.) Sensitized patients are not only more sensitive to things that should hurt, but also to ordinary touch and pressure as well. Their pain also "echoes," fading more slowly than in other people. This phenomenon is usually superimposed over other problems, but it can also occur acutely and be the primary issue, as in complex regional pain syndrome, or amplified pain syndrome, which disporportionately affects everyone.
\end{abstract}

Keywords: Pain, Nociceptors, Substance P, Kallikreins, Kinins, Opioids.

\section{Introduction}

In "PARADISE LOST" John Milton wrote that, pain is perfect misery, the worst of evils, and excessive, overturns All patience. ${ }^{1}$

Pain, inflammation and pyrexia are the most common disturbing symptom a person experiences in their life. Numerous drugs are available in the market for relieving these symptoms and which are sold over the counter. Non Steroidal Anti-Inflammatory Drugs (NSAIDs) are usually used to treat these conditions.

The widespread use of NSAIDs has meant that the adverse effects of these drugs have become increasingly prevalent. The two main adverse drug reactions (ADRs) associated with NSAIDs relate to the gastrointestinal tract and renal system. These effects are dose-dependent, and in many cases severe enough to pose the risk of ulcer perforation and upper gastrointestinal bleeding. An estimated $10-20 \%$ of patients on most commonly experience NSAIDs experience upper GIT symptoms like dyspepsia. The common side / adverse effects could be avoided by a proper prescription and health care education in view of them being sold over the counter. ${ }^{2}$

The official definition of pain endorsed by the International Association for the Study of Pain states "Pain is an unpleasant sensory and emotional experience associated with actual or potential tissue damage, or described in terms of such damage". Furthermore, the definition suggests that pain is always subjective. It is unpleasant feeling / sensation in a part or parts of the body. Management of pain must target the underlying pathophysiology and psychological factors. Pain is also beneficial symptom heralding us the involvement of a part in our body. ${ }^{3}$

Often pain is the result of "nociceptive" stimulus due to injury. We try to remove the cause of pain. Painless lesion may remain unattended, burns in Henson's, cuts in diabetes, silent infarct etc. Relief of pain by the use of opioid analgesics is an ancient pharmacotherapy still very much used today.

Arrival of a newborn is by "cry", so pain is experienced by birth. Pain is an alert due to potential damage and is protective in nature warning of health problem. Its unbridled expression often leads to considerable morbidity and suffering.

Pain is a disabling accompaniment of many medical conditions and pain control is one of the most important therapeutic priorities. Pain is a subjective experience, hard to define exactly, even though we all know that what we mean by it.

Pain has two component as (1) nociceptive component because of injury and (2) affective component because of psychological factor. They are additive to produce the clinical perception of pain. Teleologically speaking it is a desirable sensation.

Nociceptors were discovered by Charles Scott Sherrington in 1906. In earlier centuries, scientists believed that animals were like mechanical devices that transformed

*Corresponding Author: Rashmi B.R, Dept. of Physiology, A.J Institute of Allied Health Sciences, Kuntikan, Mangalore, Karnataka, India Email: rashmichandrashekar@gmail.com http://doi.org/10.18231/j.ijcaap.2019.018 
the energy of sensory stimuli into motor responses. Sherrington used many different styles of experiments to demonstrate that different types of stimulation to a nerve's receptive field led to different responses. Some intense stimuli trigger reflex withdrawal, autonomic responses and pain. The specific receptors for these intense stimuli were called nociceptors. ${ }^{4}$

Some spiritualists consider that pain purifies the mind and other consider washes out the sin of a dying person, however, the prime duty of doctor is to give relief from pain.

\section{Nociceptors (Pain Receptors)}

Nociceptors (pain receptors), detects damage occurring in tissues, which may be physical or chemical injury. ${ }^{15}$

Skin is innervated by a large number of pain fibers. Each of these arborizes into hundreds of minute free nerve endings that serve as pain receptors. The cluster of fibers from one pain fiber frequently covers an area of skin as large as 5 centimeters diameter. This area we usually call as the receptor field of that fiber. The numbers of endings are large in the center of the field but reduce towards the periphery. The arborizing fibrils overlap those from other pain fibers. Therefore, a pinprick of the skin usually stimulates endings from many different pain fibers simultaneously. When the pinprick is in the center of the receptive field of a pain fiber, degree of stimulation of that fiber is more than when it is in the periphery of the field, this is due to the number of free nerve endings in the middle of the field is much greater than at the periphery. ${ }^{15}$

\section{Types of pain and their qualities-fast pain and slow pain}

Pain could be classified into two major types as fast pain and slow pain. Fast pain is felt within about 0.1 second after a pain stimulus is applied, whereas slow pain begins only after 1 second or more and then increases slowly in seconds to minutes. Conduction pathways for these two types of pain are different and that each of them has specific qualities.

Fast pain is also described by many alternative names, such as sharp pain, pricking pain, acute pain, electric pain etc. This type of pain is felt when a needle pierces the skin, skin cut with a knife, or in burns and felt at point where the body receives. Fast-sharp pain is usually not felt in deeper tissues or organs.

Slow pain is also described by many names, such as slow burning sensation, aching pain, throbbing pain, nauseous pain, dull aching pain, chronic pain etc. This type of pain is usually associated with tissue destruction. It can lead to prolonged, unbearable pain. This could occur in skin and in any deep tissue or organ. ${ }^{15}$

\section{Pain receptors and their stimulation}

Pain receptors in the skin and other tissues are all free nerve endings. They are widespread in the superficial layers of the skin as well as in certain internal tissues, such as the periosteum, arterial walls, joint surfaces, and the falx and tentorium in the cranial vault. Most other deep tissues are only sparsely supplied with pain endings; nevertheless, any widespread tissue damage can summate to cause the slowchronic-aching type of pain in most of these areas. ${ }^{15}$

\section{Activation of nociceptors}

Pain can be elicited by multiple types of stimuli. They are classified as mechanical, thermal, and chemical pain stimuli. Usually, fast pain is elicited due to mechanical or thermal types of stimuli, whereas slow pain can be elicited by all the three types.

Chemicals that excite pain are bradykinin, serotonin, histamine, potassium ions, acids, acetylcholine, and proteolytic enzymes. Prostaglandins and substance $\mathrm{P}$ also play a role in the sensitivity of nerve endings but have no direct role. The chemical substances are especially important in stimulating the slow, type of pain that occurs after tissue injury. ${ }^{15}$

\section{Dual pathways for transmission of pain signals into the central nervous system}

Pain receptors are free nerve endings. Pain signals are transmitted to the CNS by two pathways, fast-sharp pain pathway and a slow-chronic pain pathway and hence pain classified.

The fast sharp pain signals are elicited by either mechanical or thermal pain stimuli; they are transmitted in the peripheral nerves to the spinal cord by small type $A \delta$ fibers at velocities between 6 and $30 \mathrm{~m} / \mathrm{sec}$. Conversely, the slow-chronic type of pain is elicited mostly by chemical stimuli but sometimes by persisting mechanical or thermal stimuli. This slow chronic pain is transmitted to the spinal cord by type $C$ fibers at velocities between 0.5 and $2 \mathrm{~m} / \mathrm{sec}$. Because of this dual system of pain innervations, a sudden painful stimulus often gives a "double" pain sensation: a fast-sharp pain that is transmitted to the brain by the $A \delta$ fiber pathway followed a second or so later by a slow pain that is transmitted by the $\mathrm{C}$ fiber pathway. The sharp pain heralds the person rapidly of a damaging influence and, therefore, plays an important role in making the person react immediately to remove himself or herself from the stimulus. The slow pain intensity tends to become more over a period of time. It is now, drugs play a part in relieving the pain. Pain sensation eventually produces the intolerable suffering makes the person keep trying to relieve the cause of the pain. On entering the spinal cord from the dorsal spinal roots, the pain fibers terminate on relay neurons in the dorsal horns. Here again, there are two systems for processing the pain signals on their way to the brain.

Dual pain pathways in the cord and brain stem-the neospinothalamic tract and the paleospinothalamic tract On entering the spinal cord, the pain signals take two pathways to the brain, through (1) the neospinothalamic tract and (2) the paleospinothalamic tract. 


\section{Neospinothalamic tract for fast pain}

The fast type $A \delta$ pain fibers transmit mainly mechanical and acute thermal pain. They terminate mainly in lamina I (lamina marginalis) of the dorsal horns and there excite second-order neurons of the neospinothalamic tract. These give rise to long fibers that cross immediately to the opposite side of the cord through the anterior commissure and then turn upward, passing to the brain in the anterolateral columns.

\section{Termination of the neospinothalamic tract in the brain stem and thalamus}

A few fibers of the neospinothalamic tract terminate in the reticular areas of the brain stem, but most pass all the way to the thalamus without interruption, terminating in the ventrobasal complex along with the dorsal column-medial lemniscal tract for tactile sensations. A few fibers also terminate in the posterior nuclear group of the thalamus. From these thalamic areas, the signals are transmitted to other basal areas of the brain as well as to the somatosensory cortex. ${ }^{15}$

\section{Capability of the nervous system to localize fast pain in the body}

The fast-sharp type of pain can be localized much more precisely in the different parts of the body than can slowchronic pain. However, when only pain receptors are stimulated, without the simultaneous stimulation of tactile receptors, even fast pain may be poorly localized, often only within 10 centimeters or so of the stimulated area. Yet when tactile receptors that excite the dorsal column-medial lemniscal system are simultaneously stimulated, the localization can be nearly the same.

It is believed that glutamate is the neurotransmitter substance secreted in the spinal cord at the type $A \delta$ pain nerve fiber endings. This is one of the most widely used excitatory transmitters in the central nervous system, usually having duration of action lasting for only a few milliseconds. ${ }^{15}$

\section{Paleospinothalamic pathway for transmitting slow- chronic pain}

The paleospinothalamic pathway is a system which transmits pain mainly from the peripheral slow-chronic type C pain fibers; although it does transmit some signals from type A $\delta$ fibers as well. In this pathway, the peripheral fibers terminate in the spinal cord almost entirely in laminae II and III of the dorsal horns, which together are called the substantia gelatinosa, the lateral most dorsal root type C fiber.

Most of the signals then pass through one or more additional short fiber neurons within the dorsal horns themselves before entering mainly lamina $\mathrm{V}$, also in the dorsal horn. Here the terminal neurons in the series give rise to long axons that mostly join the fibers from the fast pain pathway, passing first through the anterior commissure to the opposite side of the cord, then upward to the brain in the anterolateral pathway. ${ }^{15}$

\section{Substance p, the probable slow-chronic neurotransmitter of type $c$ nerve endings}

Type $\mathrm{C}$ pain fiber terminals entering the spinal cord secrete both glutamate transmitter and substance $\mathrm{P}$ transmitter. The glutamate transmitter is released and lasts for only a few milliseconds. Substance P is released much more slowly, building up in concentration over a period of seconds or even minutes. In fact, it has been postulated that the "double" pain sensation one feels after a pinprick might result partly from the fact that the glutamate transmitter gives a faster pain sensation, whereas the substance $\mathrm{P}$ transmitter gives a more lagging sensation. It is now understood that glutamate is the neurotransmitter most involved in transmitting fast pain into the central nervous system, and substance $\mathrm{P}$ is concerned with slow-chronic pain. ${ }^{15}$

Projection of the paleospinothalamic pathway (slowchronic pain signals) into the brain stem and thalamus The paleospinothalamic pathway terminates widely in the brain stem. Only one tenth to one fourth of the fibers passes all the way to the thalamus.

It is seen that, most terminate in one of three areas: (1) the reticular nuclei of the medulla, pons, and mesencephalon; (2) the tectal area of the mesencephalon deep to the superior and inferior colliculi; or (3) the periaqueductal gray region surrounding the aqueduct of Sylvius. These lower regions of the brain appear to be important for feeling the suffering types of pain, because animals whose brains have been sectioned above the mesencephalon to block pain signals from reaching the cerebrum still evince undeniable evidence of suffering when any part of the body is traumatized. From the brain stem pain areas, multiple short-fiber neurons relay the pain signals upward into the intralaminar and ventrolateral nuclei of the thalamus and into certain portions of the hypothalamus and other basal regions of the brain. ${ }^{15}$

\section{Pain suppression ("Analgesia") system in the brain and spinal cord}

The brain has capability in itself to suppress input of pain signals to the nervous system by activating a pain control system, called an analgesia system.

The analgesia system consists of three major components: The periaqueductal gray and periventricular areas of the mesencephalon and upper pons surround the aqueduct of Sylvius and portions of the third and fourth ventricles. Neurons from these areas send signals to the raphe magnus nucleus, a thin midline nucleus located in the lower pons and upper medulla, and the nucleus reticularis paragigantocellularis, located laterally in the medulla. From these nuclei, second-order signals are transmitted down the dorsolateral columns in the spinal cord to a pain inhibitory complex located in the dorsal horns of the spinal cord. At 
this point, the analgesia signals can block the pain before it is relayed to the brain. ${ }^{15}$

Electrical stimulation either in the periaqueductal gray area or in the raphe magnus nucleus can suppress many strong pain signals entering by way of the dorsal spinal roots. Also, stimulation of areas at still higher levels of the brain that excite the periaqueductal gray area can also suppress pain. Some of these areas are the periventricular nuclei in the hypothalamus, lying adjacent to the third ventricle, and to a lesser extent, the medial forebrain bundle, also in the hypothalamus. Several transmitter substances are involved in the analgesia system; especially involved are enkephalin and serotonin. Many nerve fibers derived from the periventricular nuclei and from the periaqueductal gray area secrete enkephalin at their endings. ${ }^{15}$

The endings of many fibers in the raphe magnus nucleus release enkephalin when stimulated. Fibers originating in this area send signals to the dorsal horns of the spinal cord to secrete serotonin at their endings. The serotonin causes local cord neurons to secrete enkephalin. The enkephalin is believed to cause both presynaptic and postsynaptic inhibition of incoming type $\mathrm{C}$ and type $\mathrm{A} \delta$ pain fibers where they synapse in the dorsal horns. Thus, the analgesia system can block pain signals at the initial entry point to the spinal cord. In fact, it can also block many local cord reflexes that result from pain signals, especially withdrawal reflexes. ${ }^{15}$

\section{Brain's opiate system-endorphins and enkephalins}

More than three decades ago it was discovered that injection of minute quantities of morphine either into the periventricular nucleus around the third ventricle or into the periaqueductal gray area of the brain stem causes an extreme degree of analgesia. In subsequent studies, it has been found that morphine-like agents, mainly the opiates, also act at many other points in the analgesia system, including the dorsal horns of the spinal cord. Because most drugs that alter excitability of neurons do so by acting on synaptic receptors, it was assumed that the "morphine receptors" of the analgesia system must be receptors for some morphine-like neurotransmitter that is naturally secreted in the brain. Therefore, an extensive search was undertaken for the natural opiate of the brain. About a dozen such opiate-like substances have now been found at different points of the nervous system; all are breakdown products of three large protein molecules: proopiomelanocortin, proenkephalin, and prodynorphin. Among the more important of these opiate-like substances are $\beta$-endorphin, met-enkephalin, leuenkephalin, and dynorphin.

The two enkephalins are found in the brain stem and spinal cord, in the portions of the analgesia system, and $\beta$ endorphin is present in both the hypothalamus and the pituitary gland. Dynorphin is found mainly in the same areas as the enkephalins, but in much lower quantities. Thus, although the fine details of the brain's opiate system are not understood, activation of the analgesia system by nervous signals entering the periaqueductal gray and periventricular areas, or inactivation of pain pathways by morphine-like drugs, can almost totally suppress many pain signals entering through the peripheral nerves. ${ }^{15}$

\section{Transient receptor potential channel}

TRPV1 (transient receptor potential vanilloid type 1 receptor) was initially identified as the receptor for capsaicin, the pungent ingredient in peppers. It is a nonselective cation channel, predominantly expressed by sensory neurons. TRPV1 is activated by a diverse range of chemical ligands (capsaicin, resiniferatoxin [RTX], endogenous lipid anandamide, and other endogenous capsaicin-like substances), as well as protons, heat, and it can behave as an integrator of the effects of many painproducing agents. ${ }^{16-18}$

Ten years after the cloning of TRPV1, several other TRPs have been described in dorsal root ganglia: TRPV2, TRPV3, TRPV4, TRPA1, and TRPM8. These channels are emerging as sensory transducers that may participate in the generation of pain evoked by chemical, thermal and mechanical stimuli. Interaction ("teamwork") between TRP channels was suggested for their role in many pain mechanisms. TRPV1 is the first described and most studied TRP receptor that may play both pronociceptive and protective roles in the various models of inflammatory and neuropathic pain syndromes. There are indications that TRPV1 may be sensitized and upregulated during inflammation and in other disease states. TRPV1 is present not only in primary sensory neurons but also in various brain nuclei, throughout the whole neuraxis, and is also expressed in non-neuronal tissues such as the epidermis (keratinocytes), urothelium, bronchial epithelium, alveolar cells, mast cells, fibroblasts and smooth muscle. Therefore, agents acting via TRPV1 may have therapeutic values in addition to pain relief. ${ }^{16-18}$

\section{TRPV1 Structure}

TRPV1 has six transmembrane helices for sensing stimuli, with the cation pore forming between domains 5 and 6 , where glycosylation occurs on Asn604. The regions that are critical for sensing heat, protons (acid), and ligands are distinct from one another. Six ankyrin repeats, attached at the N-terminal to the transmembrane complex, mediate protein binding, 1 and TRPV1 has been reported to interact with calmodulin, tubulin, AKAP150, protein kinase D (PRKCM), PKC $\gamma, \quad c$-src kinase (CSK), neurotrophin tyrosine kinase receptor 1 (NTRK1), a phosphoinositidebinding protein (Pirt), and possibly TRVP3. Of its 839 amino acids, TRPV1 reportedly can be phosphorylated by Ser/Thr kinases on residues $117,145,371,502,705,775$, 801, and 821 (numbers for human TRPV1).

Vanilloid agonists binding to TRPV1 open the channel pore and lead to cation, predominantly calcium, influx that causes membrane depolarization. When membrane depolarization reaches the threshold level, action potentials are generated and may be perceived as itch or pain. 
Vanilloid agonists release a variety of proinflammatory neuropeptides (calcitonin gene-related peptide [CGRP], cholecystokinin $[\mathrm{CCK}]$, substance $\mathrm{P}[\mathrm{SP}]$, galanin, and somatostatin) from sensory nerve endings and initiate neurogenic inflammation. Excitation of sensory neurons by vanilloid agonists is followed by a refractory state in which neurons do not respond to various stimuli, including a subsequent vanilloid challenge. This process starts with receptor desensitization (rapid loss of activity of the receptor occupied by an agonist) and leads to inactivation of vanilloid-sensitive neurons. The vanilloid-induced inactivation may be reversible or irreversible depending on the dose of agonist and its administration site. These different states induced by vanilloid agonists have led to their use for opposite aims: to induce pain (in experiments) and to relieve it. Inactivation of vanilloid-sensitive neurons is most likely not a single, well-defined biochemical process but rather a cascade of events that starts with TRPV1 activation, with calcium entry from outside the cell as well as calcium release from endoplasmic stores playing a leading role. ${ }^{19-23}$

\section{Regulation through phosphorylation}

Functionally, the ability of TRPV1 to be activated depends on a complicated balance of phosphorylation and dephosphorylation signals. In general, phosphorylation serves to sensitize the receptor for activation, whereas dephosphorylation makes the receptor non-responsive to stimuli (heat, acid, capsaicin, etc.). Numerous agonists which activate Gaq-protein coupled receptors sensitize TRPV1. These include proteases through protease-activated receptors, ATP through purinergic receptors, bradykinin through B1 and B2, and endothelin through ETAR. These receptors activate PLC $\beta$, which cleaves phosphatidylinositol bisphosphate $\left(\mathrm{PIP}_{2}\right)$ to produce inositol trisphosphate and DAG, leading to the activation of protein kinase $\mathrm{C}$ (PKC). In many of these studies, the isoform of PKC that is activated has been demonstrated to be PKC- $\varepsilon$, which requires DAG but not $\mathrm{Ca} 2+$ for activation. This kinase has been shown to phosphorylate TRPV1 on Ser502 and Ser801.

Another group of agonists can potentiate signaling through TRPV1 by activating protein kinase A (PKA). These agonists have receptors that are Gas-protein coupled, which triggers the synthesis of cAMP by adenylyl cyclase, with cAMP activating PKA. Agonists that have been demonstrated to do this include $\mathrm{PGE}_{2}$, which elevates cAMP through $\mathrm{EP}_{2}$ and $\mathrm{EP}_{4}$, prostacyclin, serotonin and 5hydroxytryptamine. At least seven residues of TRPV1 are phosphorylated by PKA in vitro; however, targets that have been shown to be physiologically relevant include Ser117, Thr145, Thr371, and Ser502. Atrial natriuretic peptide, which uses receptors that increase cGMP to signal through protein kinase $\mathrm{G}$ (PKG), raise TRPV1 activity, although it is not known whether PKG phosphorylates TRPV1. Also, it is not clear whether ligands that activate Gai-protein coupled receptors, which suppress cAMP production, alter TRPV1 activity. ${ }^{24-26}$

\section{TRVP1 Antagonists}

The other approach to find new therapeutic strategies involving TRPV1 is with the use of TRPV1 antagonists. The TRPV1-agonist-based analgesia is very different from that provided with TRPV1 antagonists. TRPV1 agonists silence the whole nerve terminal, whereas antagonists selectively block TRPV1 receptor. Interest in TRPV1 antagonists was significantly increased by recent advances suggesting that there are endogenous TRPV1 activators or "endovanilloids" and that anandamide may be one such compound. ${ }^{27-30}$

\section{Kallikreins and Kinins}

The kinins are powerful algesic agents that cause an intense burning pain when applied to the exposed base of a blister. Bradykinin excites primary sensory neurons and provokes the release of neuropeptides such as substance $P$, neurokinin $\mathrm{A}$, and calcitonin gene-related peptide. Although there is overlap, B2 receptors generally mediate acute bradykinin algesia, whereas the pain of chronic inflammation appears to involve increased numbers of B1 receptors. ${ }^{31}$

\section{History of opioids}

It is hard to decide when and where the opium poppy was first cultivated. It may have been grown for its seeds before people discovered how to prepare mekonion from the leaves and fruits of the plant or opium (from "opos," the Greek word for juice) from the liquid that appears on the unripe seed capsule when it is notched.

The use of written records to decipher the early history of opium use and abuse is hard because descriptions of drugs by ancient authors are often ambiguous. The preparation described by Homer-given by Helen, the daughter of Zeus, to Telemachus and his friends to help them forget their grief over Odysseus' absence was attributed to Homer's imagination by Theophrastus (300 B.C.) who was himself aware of the method used to produce opium. Other writers (e.g., Diskourides, A.D. 60) have argued that the drug alluded to by Homer contained henbane, the active ingredient of which is scopolamine. Most modem pharmacologists including Schmiedeberg and Lewin feel that Helen administered opium to the men. Indeed, Kritikos and Papadaki have suggested that Telemachus may not have experienced any of the toxic effects of opium because he and his contemporaries used it habitually. ${ }^{32-34}$

Despite difficulties in interpreting ancient writings and archeological data, a picture of opium use in antiquity does emerge from them. There is general agreement that the Sumerians, who inhabited what, is today Iraq, cultivated poppies and isolated opium from their seed capsules at the end of the third millenium B.C. They called opium "gil," the word for joy, and the poppy "hul gil," plant of joy. It appears that opium spread from Sumeria to the remainder of 
the old world. At first opium may have been employed as a euphoriant in religious rituals, taken by mouth or inhaled from heated vessels. ${ }^{35}$ Knowledge of its use may initially have been confined to priests representing gods who healed the sick and gods of death as well. It was given along with hemlock to put people quickly and painlessly to death, and it came to be used medicinally. The Ebers Papyrus (ca. 1500 B.C.), for example, includes the following description of a "remedy to prevent excessive crying of children. "Spenn", the grains of the spenn (poppy)-plant, with excretions of flies found on the wall, strained to a pulp, passed through a sieve and administered on four successive days. The crying will stop at once." This remedy and others containing opium (such as spongia somnifera, sponges soaked in opium used to relieve pain during surgery) were dangerous because they varied in potency and rate of absorbance. Consequently, many physicians were wary of using them. Most authors agree that, as early as the eighth century A.D., Arab traders brought opium to India and China and that between the tenth and thirteenth centuries opium made its way from Asia to all parts of Europe. ${ }^{36-38}$

British, later joined by the French, forced the Chinese to permit opium trade and consumption. In 1806, Serturmer isolated the active ingredient in opium and named it morphine after the god of dreams, Mor- pheus. (Codeine was isolated from opium a few years later). After the invention of the hypordermic syringe and hollow needle in the 1850 s, morphine began to be used for minor surgical procedures, for postoperative and chronic pain, and as an adjunct to general anesthetics. In fact, it was Claude Bernard who first investigated the use of morphine for premedicating experimental animals. He found that it reduced the amount of chloroform needed to produce anesthesia. ${ }^{39,40}$

Unfortunately, morphine had just as much potential for abuse as opium and was not terribly safe to use either. Consequently, a great deal of energy was spent trying to develop a safer, more efficacious, nonaddicting opiate. In 1898 , heroin was synthesized and pronounced to be more potent than morphine and free from abuse liability. This was the first of several such claims for novel opiates. To date, none has proven valid. In 1939, the search for a synthetic substitute for atropine culminated serendipitously in the discovery of meperidine, the first opiate with a structure altogether different from that of morphine. This was followed in 1946 by the synthesis of methadone another structurally unrelated compound with pharmacological properties similar to those of morphine. ${ }^{41,42}$

\section{Conclusion}

Pain is a symptom and not a disease. Many factors involve in generation of pain and understanding each factor for causation of pain is difficult task. However, it is the psychological pain with stress which is more painful than physical pain which lacks less evidence physically. So, further researches are going on to find more insight in to these aspects.

\section{Source of Funding}

None.

\section{Conflict of Interest}

None.

\section{References}

1. Paradise Lost (published1667), bk.6, 1.461^3.

2. Green, Ga. "Understanding NSAIDs: from aspirin to COX-2". Clin Cornerstone 2003;3(5):50-60.

3. Bonica JJ. The need of taxonomy. Pain 1979; 6(3): 247-48.

4. Sherrington C. The Integrative Action of the Nervous System. Oxford: Oxford University Press; 1906.

5. Noah T. Ashley, Zachary M. Weil, Randy J. Nelson. Inflammation: Mechanisms, Costs, and Natural Variation. Annu Rev Ecol, Evol, Systematics 2012;43:385-406.

6. Axelrod YK, Diringer MN. "Temperature management in acute neurologic disorders". Neurol Clin 2008;26(2):585-603.

7. Karakitsos D, Karabinis A. "Hypothermia therapy after traumatic brain injury in children". $N$ Engl J Med 2008:359(11):1179-80.

8. Guyton AC. Somatic Sensations: II. Pain, Headache, and Thermal sensations. In: Guyton AC, Hall JE. editors. Textbook of Medical Physiology. $11^{\text {th }}$ ed. China: Elsevier Saunders; 2006.p.894.

9. Benzinger, T. H., Heat Regulation: homeostasis of central temperature in man., Physiol Rev 1969;49:671.

10. S. Dalal and D. S. Zhukovsky, "Pathophysiology and management of fever," J Supportive Oncol 2006;4(1):9-16.

11. Humber JM. The role of complementary and alternative medicine: accommodating pluralism. J Am Med Assoc 2002;288:1655-6.

12. Hankey A. CAM modalities can stimulate advances in theoretical biology. Evid Based Complement Alternat Med 2005;2:5-12.

13. Khalsa KP. The practitioner's perspective: introduction to Ayurvedic herbalism. J Herb Pharmacother 2007;7(3-4):12942.

14. Samy RP, Pushparaj PN, and Gopalakrishnakone P. A compilation of Bioactive Compounds from Ayurveda Bioinformation 2008; 3(3): 100-10.

15. Guyton AC. Somatic Sensations: II. Pain, Headache, and Thermal sensations. In: Guyton AC, Hall JE. editors. Textbook of Medical Physiology. $11^{\text {th }}$ ed. China: Elsevier Saunders; 2006.p.572-603.

16. Caterina M, Schumacher MA, Tominaga M, Rosen TA, Levine JD, Julius D. The capsaicin receptor: a heat-activated ion channel in the pain pathway. Nature 1997;389:816-24.

17. Szallasi A, Blumberg PM. Vanilloid (capsaicin) receptors and mechanisms. Pharmacol Rev 1999;51:159-212.

18. Szallasi A, Cortright DN, Blum CA, Eid SR. The vanilloid receptor TRPV1: 10 years from channel cloning to antagonist proof-of-concept. Nat Rev Drug Discov 2007;6:357-72.

19. Jon D. Levine, Nicole Alessandri-Haber. TRP channels: Targets for the relief of pain. Biochimt Biophys Acta (BBA) Molecular Basis Dis 2007;1722(8):989-1003.

20. Eun Sy, Jung SJ, Park YK, Kwak J, Kim SJ, Kim J et al. Effects of capsaicin on $\mathrm{Ca}^{2+}$ release from the intracellular $\mathrm{Ca}^{2+}$ stores in the dorsal root ganglion cells of adult rats. Biochem Biophys Res Commun 2001;285:1114-20.

21. Marshall IC, Owen DE, Cripps TV, Davis JB, McNulty S, Smart D. Activation of vanilloid receptor 1 by resiniferatoxin mobilizes calcium from inositol 1, 4, 5-trisphosphate-sensitive stores. Br J Pharmacol 2003;138:172-6. 
22. Lishko P.V, E. Procko, X. Jin, C.B. Phelps, and R. Gaudet. The ankyrin repeats of TRPV1 bind multiple ligands and modulate channel sensitivity. Neuron 2007;54:905-18.

23. Mohapatra D.P. and Nau C. Regulation of $\mathrm{Ca}^{2+}$ - dependant desensitization in the vanillioid receptor TRPV1 by calcineurin cAMP- dependant protein kinase. J Biol Chem 2005;280:13424-32.

24. Xuming Zhang, Jiehong Huang, Peter A McNaughton. NGF rapidly increases membrane expression of TRPV1 heat-gated ion channels. EMBO J 2005;24(24):4211-23.

25. Lukacs V, Thyagarajan B, Varnai P, Balla A, Balla T, Rohacs T et al. Dual Regulation of TRPV1 by Phosphoinositides. $J$ Neurosci 2007;27(26):7070-80.

26. Szallasi A, Cortright D.N, Blum C.A, and Eid S.R. Nat. Rev. The vanilloid receptor TRPV1: 10 years from channel cloning to antagonist proof-of-concept. Drug Discov 2007;6:357-72.

27. Ross RA. Anandamide and vanilloid TRPV1 receptors. Br J Pharmacol 2003;140:790-801.

28. Szallasi A, DiMarzo V. New perspectives on enigmatic vanilloid receptors. Trend Neurosci 2000;23:491-7.

29. Levine JD, Alessandri-Haber N. TRP channels: Targets for the relief of pain. Biochim Biophys Acta 2007;1772(8):989-1003.

30. Szallasi A, Appendino G. Vanilloid receptor TRPV1 antagonists as the next generation of painkillers. Are we putting the cart before the horse? J Med Chem 2004;47:271723.

31. Randal A, Skidgel, Allen P. Kalpan, Ervin G. Erdos. Histamine, Bradykinin, and Their antagonist. In: Brunton LL. editor. Goodman \& Gillman's Manual of Pharmacology \& Therapeutics. $11^{\text {th }}$ ed. New Delhi: McGraw-Hill; 2008.p.413

32. Schmiedeberg, O.Schr. Wiss. Gesellsch. (Strassburg). 1918;36:1-29.
33. Lewin L. Phantastica, narcotic and stimulating drugs. New York, EP Dutton \& Co; 1964:193.

34. Kritikos, P. G. \& Papadaki, S. P. The History of the Poppy and of Opium and Their Expansion in Antiquity in the Eastern Mediterranean. Bull Narc 1967;19(3):17-38.

35. Kritikos, P. G. \& Papadaki, S. P. The History of the Poppy and of Opium and Their Expansion in Antiquity in the Eastern Mediterranean . Bull Narc 1967;19(4):5-10.

36. Lubec G, Holaubek J, Feldl C, Lubec B. \& Strouhal, Use of silk in ancient. Egypt. Nature (London). 1993;362:25.

37. Dwarakanath, S. C. Use of opium and cannabis in the traditional system of medicine in india. Bull. Narc.1965;17(1):15-9.

38. Fort, J. Giver of delight or liberator of sin: Drug use and "addiction" in Asia. Bull Narc 1965;17(3):1-11.

39. Serturner, F. W. A. "Darstellung der reinen Mohnsäure (Opiumsäure) nebst. Journal der Pharmacie fuer Aerzte und Apotheker. J Pharm f Arzte Apoth Chem 1806;14:47-93.

40. Serturner, F. W. A. Untersuchungen über die wasserfrei organischen Säuren. Gilbert's Ann. d. Physik 1817;25:56-89.

41. Eisleb, O.; Schaumann, O.: Dolantin, ein neuartiges Spasmoly- tikum and Ana. Dtsch Med Wochenschr 1939;65:967-8.

42. Scott, C. C., Robbins EB, Chen, K. K. Comparison of Some New Analgesic Compounds. J Pharmacol Exp Ther 1946;87:63-71.

How to cite this article: Rashmi BR, Ebrahim NKC. A review on pain and its signals modulation by body. Int $J$ Comprehensive Adv Pharmacol 2019;4(3):84-90. 